\title{
Hybrid lipids, peptides, and lymphocytes: new era in type 1 diabetes research
}

\author{
Abdel Rahim A. Hamad, ${ }^{1}$ Mohanraj Sadasivam, ${ }^{1}$ and Hamid Rabb ${ }^{2}$ \\ 'Department of Pathology and ${ }^{2}$ Department of Medicine, Johns Hopkins University, Baltimore, Maryland, USA.
}

Type 1 diabetes (T1D) results from autoimmune destruction of insulinproducing $\beta$ cells in islets of Langerhans. Many genetic and immunological insights into autoimmune disease pathogenesis were initially uncovered in the context of T1D and facilitated by preclinical studies using the nonobese diabetic (NOD) mouse model. Recently, the study of T1D has led to the discovery of fatty acid esters of hydroxyl fatty acids (FAHFAs), which are naturally occurring hybrid peptides that modulate inflammation and diabetes pathogenesis, and a hybrid lymphocyte that expresses both $B$ and T cell receptors. Palmitic acid esters of hydroxy stearic acids (PAHSAs) are the most extensively studied FAHFA. In this issue of the $J C I$, Syed et al. have shown that PAHSAs both attenuate autoimmune responses and promote $\beta$ cell survival in NOD mice. Given the lack of effective T1D therapies and the paucity of known side effects of PAHSAs, this lipid may have therapeutic potential for individuals at risk for or newly diagnosed with T1D.

\section{Introduction}

Type 1 diabetes (T1D) is an autoimmune disease caused by the destruction of insulin-producing $\beta$ cells by autoreactive $\mathrm{T}$ cells (1). This loss of $\beta$ cells results in insulin deficiency and impaired glucose homeostasis manifested by hyperglycemia. New cases of T1D are increasing worldwide for unknown reasons, compromising patients' quality and length of life. There is currently no cure for T1D, and insulin replacement therapy is the cornerstone of disease management (2). Despite major advances in glucose monitoring and insulin delivery techniques, many patients with T1D develop long-term complications $(2,3)$. Thus, there is an important need to develop strategies to prevent and/ or delay disease progression. Efforts to develop preventative and disease-halting approaches are being pursued in parallel but complementary tracks. For example, immunologists are focusing on develop- ing immunotherapies to curtail or remove autoreactive T cells $(4,5)$, while cell biologists are focusing on the generation of $\beta$ cells using stem cells and identifying strategies to promote $\beta$ cell repair and survival (6). The use of immunotherapy is hampered by a lack of specific targets, and broad-based immunological targeting is limited by inherent serious side effects (2). The generation of stem cell-derived $\beta$ cells and efforts to enhance repair have yet to produce clear-cut successes (7). In this issue, Syed et al. present exciting data supporting the use of palmitic acid esters of hydroxy stearic acids (PAHSAs) as a safe approach for the treatment of experimental T1D, one that regulates autoreactive $\mathrm{T}$ cells and enhances islet regeneration (8). Given the apparently few side effects associated with PAHSAs, future use of these hybrid peptides may prove beneficial for individuals at risk for or newly diagnosed with T1D.

Related Article: p. 3717

Conflict of interest: The authors have declared that no conflict of interest exists.

Copyright: @ 2019, American Society for Clinical Investigation.

Reference information: / Clin Invest. 2019;129(9):3527-3529. https://doi.org/10.1172/JCI130313.

Autoimmune destruction of pancreatic islet $\beta$ cells

The pathogenesis of autoimmune diabetes is being extensively investigated in both humans and in animal models (9). Though the exact mechanisms that underlie autoimmune destruction of $\beta$ cells remain elusive, it is generally believed that the disease process is triggered by the activation of autoreactive $\mathrm{T}$ cells by islet autoantigens, such as insulin, GAD65, IA2, and ZNT18. These autoantigens are taken up by antigen-presenting cells (APCs), including macrophages, DCs, and B cells, and then processed and presented to autoreactive $T$ cells in pancreatic draining lymph nodes (1). In addition, a public B cell receptor (BCR) has recently been shown to encode a potent autoantigen (10), x-autoantigen, which can stimulate autoreactive $\mathrm{T}$ cells in vitro as a peptide tethered in the cleft of HLA-DQ8 molecules (a T1Dpredisposing MHC class II molecule) or as a soluble antibody. Activated autoreactive $\mathrm{T}$ cells proliferate, migrate to the pancreas, and infiltrate islets, resulting in insulitis. Islet-infiltrating $\mathrm{T}$ cells are kept in check for a while, at least in mice, before they start attacking and destroying insulinproducing $\beta$ cells. A lack of key molecules, including an impaired Fas death pathway, prevents insulitis in nonobese diabetic (NOD) mice $(11,12)$. Furthermore, insulitis can remain relatively benign for varying periods of time in NOD mice before the destruction of $\beta$ cells by infiltrating $\mathrm{T}$ cells (13). The mechanisms that trigger the transition of insulitis from a benign to a destructive state are poorly understood and may include Treg deficiency (14) and/ or environmental factors.

\section{Signaling pathways that induce $\beta$ cell destruction}

Several cytokines and a number of key pathways are involved in $\beta$ cell apoptosis following attack by autoreactive lymphocytes and innate immune cells. For example, $\beta$ cells are highly sensitive to IL- $1 \beta$ and 


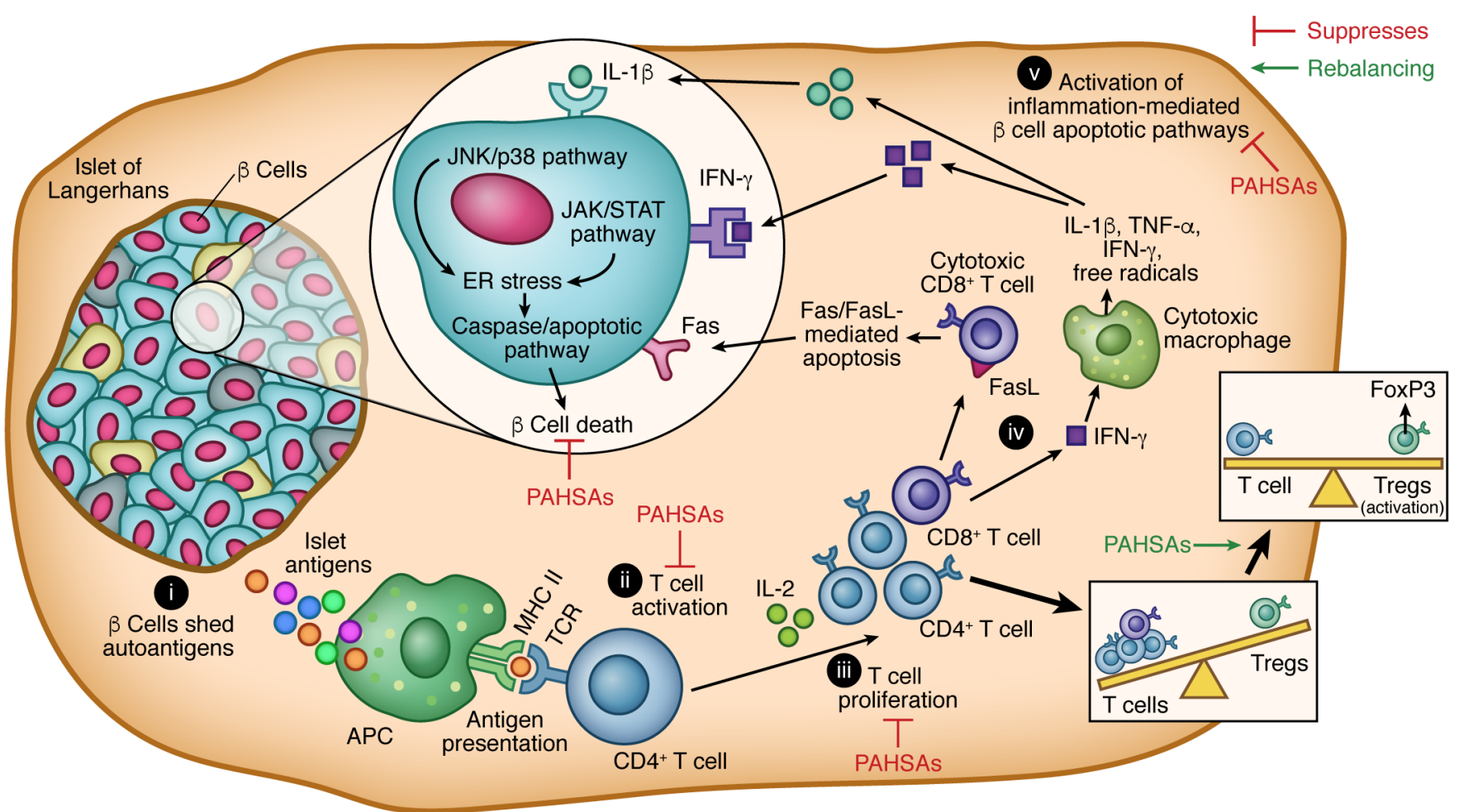

Figure 1. Key steps in the pathogenesis of T1D and sites modulated by PAHSAs. Autoimmunity is initiated by the release of islet autoantigens (i) by pancreatic $\beta$ cells that are taken up by APCs, processed, and presented in the context of MHC class molecules to autoreactive T cells (ii). Activated CD4+ T cells proliferate (iii) and release proinflammatory cytokines and then help activate cytotoxic CD8 ${ }^{+} \mathrm{T}$ cells (iv) that use different mechanisms to attack and destroy insulin-producing $\beta$ cells (v). PAHSAs attenuate T cell activation and proliferation and increase Treg activity. Further, PAHSAs attenuate cytokine-mediated $\beta$ cell apoptotic pathways, decrease $\beta$ cell destruction, and improve $\beta$ cell survival. Illustrated by Rachel Davidowitz.

IFN- $\gamma$ inflammatory cytokines (15). IL-1 $\beta$ activates the JNK/p38 MAPK pathway, and IFN- $\gamma$ activates JAK/STAT signaling, both of which initiate $\beta$ cell apoptosis. Disruption of ER homeostasis, characterized by an increase in $\mathrm{ER} \mathrm{Ca}^{2+}$ concentrations and accumulation of unfolded proteins, leads to an ER-specific stress response, followed by $\beta$ cell death. Mitochondria are key organelles for $\beta$ cell survival and function, and changes in mitochondria can trigger proapoptotic signaling, in turn leading to the activation of caspase-mediated $\beta$ cell apoptosis (Figure 1). Along with these pathways, the Fas/FasL pathway may directly initiate $\beta$ cell destruction, given that mice with impaired Fas signaling are protected from insulitis (12).

Multiple mechanisms and pathways regulate effector immune and autoimmune responses, including antiinflammatory cytokines, such as IL-10 and TGF- $\alpha$ (16), and Tregs, which are induced and maintained by lineage-specific transcription factor FoxP3 (17). Loss-of-function mutations of the FoxP3-encoding gene lead to severe fatal autoimmune disorders in humans and mice (18), indicating the importance of this factor for maintaining Treg function and homeostasis.

\section{PAHSAs increase pancreatic $\beta$ cell survival and improve experimental T1D}

Increased levels of circulating fatty acids are generally associated with insulin resistance and impaired glucose tolerance (19). PAHSAs are an endogenous lipid and the most commonly investigated form of fatty acid esters of hydroxyl fatty acids (FAHFAs). Previous studies have shown that PAHSAs improve insulin sensitivity and reduce insulin resistance (20). In mice, PAHSA administration lowers glycemia, improves insulin sensitivity, and enhances glucose tolerance (20). Syed et al. have now demonstrated that PAHSAs attenuate autoimmune responses and promote $\beta$ cell survival in NOD mice. Administration of PAHSAs to NOD mice delayed the onset of T1D and markedly reduced T1D incidence. Moreover, PAHSAs reduced
$\mathrm{T}$ and $\mathrm{B}$ cell infiltration and activation, while increasing Treg activation in NOD mice pancreata. PAHSAs promoted $\beta$ cell proliferation in both NOD mice and in the human pancreatic MIN6 cell line and increased $\beta$ cell numbers in NOD mice. PAHSAs attenuated cytokine-induced apoptotic and necrotic $\beta$ cell death, while increasing $\beta$ cell viability. The mechanisms that underlie these beneficial effects were shown to involve reduced ER stress responses via a reduction of JNK/MAPK pathways. Moreover, the study supported a role for glucagon-like peptide 1 (GLP-1) in mediating PAHSA-associated improvements in insulin secretion.

The findings of Syed et al. have potential for translation to humans (8) given the mild reported side effects of PAHSAs; however, many questions remain unanswered. The mechanisms by which PAHSAs regulate the immune system in T1D or $\beta$ cell function are incompletely understood. It is still unclear how PAHSAs prevent ER stress. PAHSAs have been demonstrated to affect both the immune system and $\beta$ cells, but 
which target is more important and what are the relationships between the two? It is notable that a different study showed a limited or no effective response to repeated injections of PAHSAs into mice (21). Although different methodologies may well explain this discrepancy, additional confirmation that PAHSAs are indeed modulators of autoimmune responses in T1D is necessary. Future studies could explore the direct effects of PAHSA-treated Tregs on autoreactive $\mathrm{T}$ cell suppression. A detailed mechanistic study involving PAHSA-mediated suppression of ER stress would be useful. These and future studies lay the groundwork for human trials to evaluate PAHSA treatment for T1D.

\section{Acknowledgments}

The authors are supported by NIH grants R01-DK104662 and R0 AI099027.

Address correspondence to: Abdel Rahim Hamad, Johns Hopkins University School of Medicine, Ross 66G, 720 Rutland Avenue, Baltimore Maryland 21205, USA. Phone: 410.614.3021; Email: ahamad@jhmi.edu.

1. Pugliese A. Autoreactive T cells in type 1 diabetes. J Clin Invest. 2017;127(8):2881-2891.

2. Pettus J, Von Herrath M. The shifting paradigm of a "cure" for type 1 diabetes: is technology replacing immune-based therapies? Acta Diabe- tol. 2018;55(2):117-120.

3. Hamad AR, Ahmed R, Donner T, Fousteri G. B cell-targeted immunotherapy for type 1 diabetes: what can make it work? Discov Med. 2016;21(115):213-219.

4. Ramirez L, Hamad AR. From non-obese diabetic to network for the pancreatic organ donor with diabetes: new heights in type 1 diabetes research. World J Diabetes. 2015;6(16):1309-1311.

5 . Herold KC, et al. An anti-CD3 antibody, teplizumab, in relatives at risk for type 1 diabetes [published online ahead of print June 9, 2019]. NEngl JMed. https://doi.org/10.1056/ NEJMoa1902226.

6. Zhong F, Jiang Y. Endogenous pancreatic $\beta$ cell regeneration: a potential strategy for the recovery of $\beta$ cell deficiency in diabetes. Front Endocrinol (Lausanne). 2019;10:101.

7. Cito M, Pellegrini S, Piemonti L, Sordi V. The potential and challenges of alternative sources of $\beta$ cells for the cure of type 1 diabetes. Endocr Connect. 2018;7(3):R114-R125.

8. Syed I, et al. PAHSAs attenuate immune responses and promote $\beta$ cell survival in autoimmune diabetic mice. J Clin Invest. 2019;129(9):3717-3731.

9. O'Kell AL, et al. Comparative pathogenesis of autoimmune diabetes in humans, NOD mice, and canines: has a valuable animal model of type 1 diabetes been overlooked? Diabetes. 2017;66(6):1443-1452.

10. Ahmed R, et al. A public BCR present in a unique dual-receptor-expressing lymphocyte from type 1 diabetes patients encodes a potent $\mathrm{T}$ cell autoantigen. Cell. 2019;177(6):1583-1599.e16.

11. Saxena A, Yagita H, Donner TW, Hamad ARA. Expansion of FasL-expressing $\mathrm{CD}^{+} \mathrm{B}$ cells in type 1 diabetes patients. Front Immunol. 2017;8:402.
12. Xiao Z, et al. Inhibition of Fas ligand in NOD mice unmasks a protective role for IL-10 against insulitis development. Am J Pathol. 2011;179(2):725-732.

13. André I, Gonzalez A, Wang B, Katz J, Benoist C, Mathis D. Checkpoints in the progression of autoimmune disease: lessons from diabetes models. Proc Natl Acad Sci U S A. 1996;93(6):2260-2263.

14. Lindley S, Dayan CM, Bishop A, Roep BO, Peakman M, Tree TI. Defective suppressor function in CD4(+)CD25(+) T-cells from patients with type 1 diabetes. Diabetes. 2005;54(1):92-99.

15. Cnop M, Welsh N, Jonas JC, Jörns A, Lenzen S, Eizirik DL. Mechanisms of pancreatic beta-cell death in type 1 and type 2 diabetes: many differences, few similarities. Diabetes. 2005; 54(Suppl 2):S97-107.

16. Bluestone JA, Tang Q. $\mathrm{T}_{\text {reg }}$ cells-the next frontier of cell therapy. Science. 2018;362(6411):154-155

17. Sakaguchi S, Vignali DA, Rudensky AY, Niec RE, Waldmann H. The plasticity and stability of regulatory T cells. Nat Rev Immunol. 2013;13(6):461-467.

18. Wållberg M, Cooke A. Immune mechanisms in type 1 diabetes. Trends Immunol. 2013;34(12):583-591.

19. Boden G, Shulman GI. Free fatty acids in obesity and type 2 diabetes: defining their role in the development of insulin resistance and beta-cell dysfunction. Eur JClin Invest. 2002;32(Suppl 3):14-23.

20. Yore MM, et al. Discovery of a class of endogenous mammalian lipids with antidiabetic and anti-inflammatory effects. Cell. 2014;159(2):318-332.

21. Pflimlin E, et al. Acute and repeated treatment with 5-PAHSA or 9-PAHSA isomers does not improve glucose control in mice. Cell Metab. 2018;28(2):217-227.e13. 